\title{
Introduction to the level-set full field modeling of laths spheroidization phenomenon in $\alpha / \beta$ titanium alloys
}

\author{
D. Polychronopoulou, ${ }^{1, a}$, N. Bozzolo ${ }^{1}$, D. Pino Muñoz ${ }^{1}$, J. Bruchon ${ }^{2}$, M.Shakoor ${ }^{1}$, Y. Millet ${ }^{3}$, C. Dumont ${ }^{4}$, I. Freiherr von \\ Thüngen $^{5}$, R. Besnard ${ }^{6}$ and $M$. Bernacki ${ }^{1}$ \\ ${ }^{1}$ MINES ParisTech, PSL Research University, CEMEF - Centre de mise en forme des matériaux, CNRS UMR 7635, CS 10207, rue \\ Claude Daunesse, 06904 Sophia Antipolis Cedex, France \\ ${ }^{2}$ École Nationale Supérieure des Mines de Saint-Étienne. Centre Sciences des Matériaux et des Structures. Département Mécanique \\ et Procédés d'Elaboration. 158 cours Fauriel, 42023 Saint-Etienne Cedex 02, France \\ ${ }^{3}$ Timet Savoie, 62 avenue Paul Girod, 73400 Ugine, France \\ ${ }^{4}$ Aubert \& Duval, rue des Villas, 63770 Les Ancizes-Comps, France \\ ${ }^{5}$ Safran, SafranTech - Pôle Matériaux et procédés, Rue des Jeunes Bois, Châteaufort, CS 80112, 78772 Magny-Les-Hameaux, France \\ ${ }^{6}$ CEA Valduc, 21120 Is-Sur-Tille, France
}

\begin{abstract}
Fragmentation of $\alpha$ lamellae and subsequent spheroidization of $\alpha$ laths in $\alpha / \beta$ titanium alloys occurring during and after deformation are well known phenomena. We will illustrate the development of a new finite element methodology to model them. This new methodology is based on a level set framework to model the deformation and the ad hoc simultaneous and/or subsequent interfaces kinetics. We will focus, at yet, on the modeling of the surface diffusion at the $\alpha / \beta$ phase interfaces and the motion by mean curvature at the $\alpha / \alpha$ grain interfaces.
\end{abstract}

\section{Introduction}

Two-phase $\alpha / \beta$ titanium alloys are materials with numerous applications in different industrial domains, mostly due to their attractive mechanical properties. They exhibit different microstructures depending on the applied thermomechanical path. Starting from an initial stable microstructure of $\alpha$ lamellae inside $\beta$ grains, the phenomenon of spheroidization can be observed during and after the microstructure deformation. More precisely, during the deformation, the fragmentation of $\alpha$ lamellae and the subsequent spheroidization of $\alpha$ laths occurs.

Spheroidization has received considerable attention due to its importance in microstructural control. The new spheroidized microstructure shows enhanced strength and ductility, so evidently, this phenomenon raises high interest for the industrial applications [1].

In this paper, we will illustrate a new finite element (FE) methodology in order to model these microstructural evolutions. The interest is focused on the first mechanisms occurring during spheroidization at the lamellae interfaces without considering the microstructure deformation modeling. The $\alpha / \alpha$ grain interfaces are introduced arbitrarily leading to surface diffusion at the $\alpha / \beta$ phase interfaces and the motion by mean curvature at the $\alpha / \alpha$ grain interfaces. In order to model efficiently this interfacial kinetics, a level set framework was introduced.

Some basic cases of surface diffusion will be detailed in order to illustrate the first steps and the numerical choices of the methodology.

\section{Describing the physical problem}

According to Semiatin [2], the first basic mechanisms occurring during hot deformation, in order to get the spheroidized microstructures, are the surface diffusion at the $\alpha / \beta$ interfaces and the motion by the mean curvature at the $\alpha / \alpha$ grain interfaces. The motivation for the formation of grooves in $\alpha$ lamellae and the consequent splitting of them, is the tendency of the different interfaces to evolve in order to minimize the total interfacial energy. Grooving is usually initiated by atomic scale processes near the region of $\alpha / \alpha$ grain boundary and $\alpha / \beta$ interface

\footnotetext{
${ }^{\mathrm{a}}$ Corresponding author: danai.polychronopoulou@mines-paristech.fr
} 
intersection. Next section illustrates the governing equations of this phenomenon.

\subsection{Motion by surface diffusion}

According to Mullins [3], in order to describe the atoms flow at the $\alpha / \beta$ interface we can consider a surface flux $\vec{j}$ :

$$
\vec{\jmath}=v \vec{v},
$$

where $v$ denotes the number of drifting atoms per unit area and $\vec{v}$ denotes the average velocity of these drifting atoms. Assuming local equilibrium we can express $\vec{v}$ with the Nerst-Einstein formula as following:

$$
\overrightarrow{\mathrm{v}}=\frac{\mathrm{D}_{\alpha \beta}}{\grave{\mathrm{k} T}} \nabla_{\mathrm{s}} \mu,
$$

where $D_{\alpha \beta}$ denotes the surface diffusivity of the $\alpha / \beta$ interface, $\mu$ the chemical potential, $\dot{k}$ the Boltzmann constant and $T$ the absolute temperature. The $\nabla_{s}$ operator corresponds to the surface gradient operator defined as the tangential component of the gradient:

$$
\nabla_{\mathrm{s}} \mu=\nabla \mu-(\nabla \mu \cdot \mathrm{n}) \mathrm{n}=\mathrm{P} \nabla \mu,
$$

with $\boldsymbol{n}$ the outward-pointing unit vector normal to the surface and $P=I-\boldsymbol{n} \otimes \boldsymbol{n}$.

By considering Eq.(1) and Eq.(2), the following equation is obtained:

$$
\overrightarrow{\mathrm{j}}=\frac{v \mathrm{D}_{\alpha \beta}}{\grave{\mathrm{k} T}} \nabla_{\mathrm{s}} \mu .
$$

Assuming that there is mass conservation, the surface motion can then be described by:

$$
\mathrm{v}_{\mathrm{n}}=-\grave{\Omega}\left(\nabla_{\mathrm{s}} \overrightarrow{\mathrm{j}}\right)
$$

where $v_{n}$ denotes the normal velocity of the surface

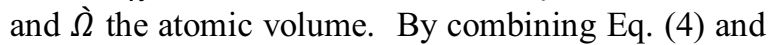
Eq. (5), we obtain:

$$
\mathrm{v}_{\mathrm{n}}=-\frac{v \grave{\Omega} \mathrm{D}_{\alpha \beta}}{\grave{\mathrm{k} T}} \Delta_{\mathrm{s}} \mu,
$$

with $\Delta_{s}=\nabla_{\mathrm{s}} \cdot \nabla_{\mathrm{s}}$ the surface Laplacian operator (or Laplace-Beltrami operator).

From Eq. (6), it is notable that the normal velocity is associated with the chemical potential of the atoms.

Considering $\kappa$ as the mean curvature (sum of the principal curvatures in 3D) and $\gamma_{\alpha \beta}$ the $\alpha / \beta$ interface energy and by ignoring the possible effects of anisotropy, the following relationship is obtained:

$$
\mu=-\gamma_{\alpha \beta} \grave{\Omega} \kappa
$$

Thanks to Eq.(6) and Eq.(7), we obtain:

$$
\mathrm{v}_{\mathrm{n}}=\frac{\gamma_{\alpha \beta} v \grave{\Omega}^{2} \mathrm{D}_{\alpha \beta}}{\grave{\mathrm{k} T}} \Delta_{\mathrm{s}} \kappa=\mathrm{B} \Delta_{\mathrm{s}} \kappa,
$$

with $B=\frac{\gamma_{\alpha \beta} v \grave{\Omega}^{2} D_{\alpha \beta}}{\dot{k} T}$, the kinetic coefficient. Eq. (8) describes the relation between the motion by surface diffusion and the surface Laplacian of the mean curvature [4] [5] [6] [7].

\subsection{Motion by mean curvature}

In order to describe precisely the surface evolution of an $\alpha$ lamella, the influence of the mean curvature should be also considered [5] [7]. The $\gamma_{\alpha \alpha}$ grain boundary energy is indeed very important for the lamellae splitting.

The grain boundary energy is given by the wellknown Gibbs-Thompson relationship where the normal velocity $v_{n}$ of the grain boundary is described proportionally to the mean curvature $\kappa$ :

$$
\mathrm{V}_{\mathrm{n}}=-\kappa \frac{\gamma_{\alpha \mathrm{a} b \check{\mathrm{v}} \grave{\Omega}}^{\grave{\mathrm{k} T}}}{\mathrm{e}^{-\frac{\Delta \mathrm{E}}{\mathrm{k} T}}}=-\mathrm{A \kappa},
$$

with $A=\frac{\gamma_{\alpha a} b \breve{v} \grave{\Omega}}{\grave{k} T} e^{-\frac{\Delta E}{\grave{k} T}}$, where $\gamma_{\alpha a}$ denotes the grain boundary energy, $b$ is the burgers vector norm associated with the hoping event, $\check{v}$ is the Debye frequency and $\Delta E$ is the difference in free energy between the two grains.

\subsection{Motion of the interfaces}

Surface diffusion and mean curvature motions are taking part simultaneously during the phenomenon of spheroidization. A global velocity combining both of these motions can then be summarized as:

$$
\mathrm{v}_{\mathrm{n}}=\mathrm{B}\left(\Delta_{\mathrm{s}} \kappa\right)-\mathrm{A} \kappa,
$$

where $B$ is defined in $\alpha / \beta$ phase interfaces and $A$ is defined in $\alpha / \alpha$ grain interfaces.

\section{Level set formulation}

A level-set model was formulated in order to deal with the topological changes at the $\alpha / \beta$ interface. The level set method was chosen due to its capability to immerse/describe/capture easily in a FE context the interfaces [8] [9] [10] [11] and also due to the fact that geometrical quantities as the mean curvature $\kappa$ and the outside normal $n$ can be obtained as: 


$$
\mathrm{n}=\frac{\nabla \varphi}{\|\nabla \varphi\|}
$$

and

with

$$
\kappa=\operatorname{div}(\boldsymbol{n})=\nabla \frac{\nabla \varphi}{\|\nabla \varphi\|^{\prime}}
$$

$$
\varphi(x, t)= \pm d(x, \Gamma(t))
$$

$\varphi$ is then defined over the domain $\Omega$ as the signed distance function to the interface $\Gamma$ of the subdomain of interest that we will denote $\Pi$.

The sign convention of Eq. (11) corresponds to a distance function negative inside $\Pi$ and positive outside.

Thus the interface velocity can be rewritten in a level set form as:

$$
\overrightarrow{\mathrm{v}}=\mathrm{v}_{\mathrm{n}} \frac{\nabla \varphi}{\|\nabla \varphi\|}=\mathrm{v}_{\mathrm{n}} \mathrm{n}=\left(\mathrm{B}\left(\Delta_{\mathrm{s}} \kappa\right)-\mathrm{A} \kappa\right) \mathrm{n},
$$

with:

$B=\left\{\begin{array}{c}\frac{\gamma_{\alpha \beta} \mathrm{v} \grave{2}^{2} D_{\alpha \beta}}{\grave{k} T}, \text { at the } \alpha / \beta \text { interface } \\ 0 \quad, \text { otherwise }\end{array}\right.$

$A=\left\{\begin{array}{cc}\frac{\gamma_{\alpha a} b \check{v} \grave{\Omega}}{\grave{k} T} e^{-\frac{\Delta E}{\grave{k} T}}, & \text { at the } \alpha / \alpha \text { interface } \\ 0, & \text { otherwise }\end{array}\right.$

It can also be proved that in the considered levelset formulation [4] [12], $v_{n}$ can be re-written as:

$$
\begin{gathered}
\mathrm{v}_{\mathrm{n}}=\mathrm{B}\left(\Delta_{\mathrm{s}} \kappa\right)-\mathrm{A} \kappa \\
=\frac{\mathrm{B}}{\|\nabla \varphi\|} \nabla \cdot(\|\nabla \varphi\| \mathrm{P} \nabla \kappa)-\mathrm{A} \kappa
\end{gathered}
$$

The velocity is then defined in the entire domain and corresponds in the vicinity of the zero level-set function of $\varphi$, i.e. $\Gamma$, to the interface velocity [4] [5].

\section{Generation of the microstructure}

As already mentioned, the $\alpha$ lamellae and their shape evolution are going to be represented with the use of a level set function. A new Representative Volume Element class was built in our fully parallel multicomponent C++ Library called CIMLib [13] and used for these numerical developments in order to describe $\alpha$ colonies in $\beta$ grains by using the level set approach.

The basic steps of this method are:

a) consideration of a given domain as a $\beta$ grain. b) division of the domain in colonies (by Voronoï tessellation for example [14]).

c) In each colony, Voronoï seeds belonging to a prescribed common axis are introduced.

d) Partition of each colony into regions, which are based to the minimum distance of the points of the domain from the Voronoï seeds of the corresponding colony. Each region consists of all the integration points closer to the corresponding seed that any other, called as Voronoï cells [14].

e) A variable $\varepsilon$ is introduced for shifting the Voronoï cells in order to manage the description of the two different phases. This variable can be defined from a distribution or from the desired volume fraction of the $\alpha$ phase.

f) All these operations can be described in a exact analytical way in the considered LS framework.

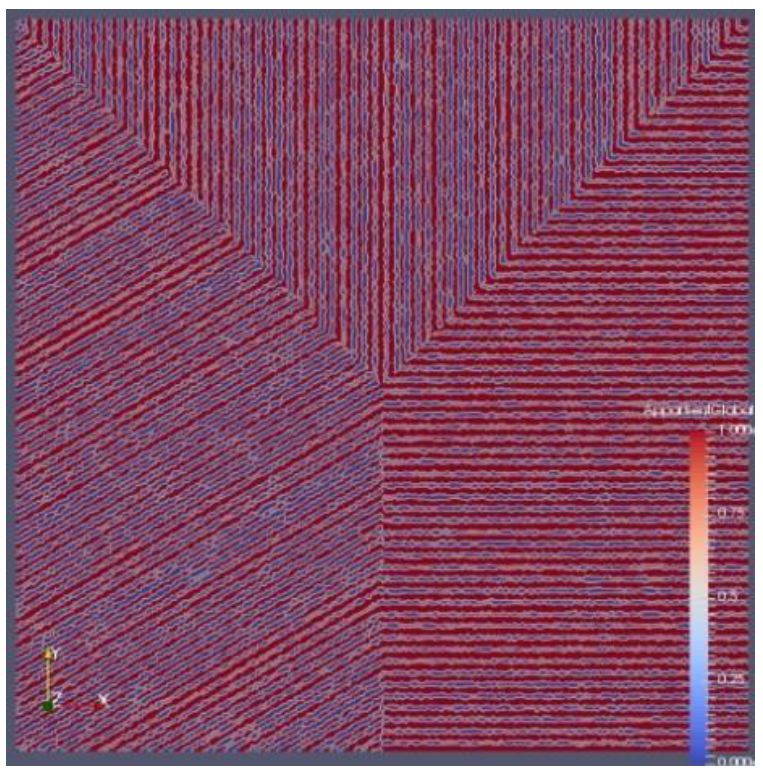

Figure 1. Representation of an initial microstructure of a $\beta$ grain with three different $\alpha$ colonies thanks to a LS approach.

Figure 1 illustrates a 2D case in a $1 \mathrm{~mm} \times 1 \mathrm{~mm}$ domain. The red regions describe the $\alpha$ lamellae and the blue regions the $\beta$ media. Furthermore, in order to emphasize the efficiency of the developed method to describe statistically the microstructure of interest, a corresponding illustrative back-scattered electrons (BSE) image obtained thanks to a scanning electron microscope (SEM) from an initial microstructure of TA-6V alloy is displayed in Figure 2. 


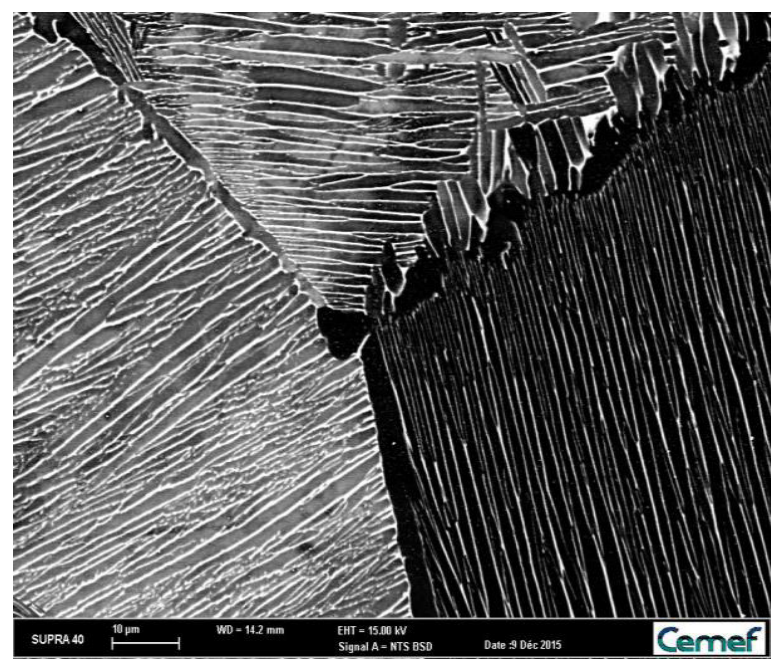

Figure 2. An initial microstructure of TA6V, with 3 different colonies.

\section{A surface diffusion methodology}

For the modelling of the induced flow from the surface diffusion mechanism at the $\alpha / \beta$ interface, a finite element solver that follows a level set method is used. At any time t, the transport velocity $\vec{v}$ is defined by:

$$
\overrightarrow{\boldsymbol{v}}=\mathrm{B}\left(\Delta_{s} \kappa\right) \boldsymbol{n}=\frac{B}{\|\nabla \varphi\|} \nabla \cdot(\|\nabla \varphi\| P \nabla \kappa) \boldsymbol{n}
$$

with $\mathrm{B}=\frac{\gamma_{\alpha \beta} v \grave{\Omega}^{2} D_{\alpha \beta}}{\grave{k} T}$. The $\mathrm{B}$ coefficient is defined as a constant and it is chosen to neglect any anisotropy concerning the interface energy and the diffusivity. Additionally, isothermal conditions are assumed. The time evolution of $\Gamma(t)$ is then obtained by solving the following convective system:

$$
\left\{\begin{array}{c}
\frac{\partial \varphi}{\partial t}+\vec{v} \cdot \overrightarrow{\nabla \varphi}=0 \\
\varphi(x, 0)=-d(x, \Gamma(0)) \chi_{\Pi}+d(x, \Gamma(0)) \chi_{\bar{\Pi}}
\end{array}\right.
$$

The interface can then be obtained at each time step as the 0 -isovalue of the distance function and the velocity is updated by following Eq.(18) before the following time step. At the following subsections, more extensive details are given for the resolution algorithm.

\subsection{Surface diffusion velocity identification and transport resolution}

The methodology used to obtain the surface diffusion velocity is based on the finite element based strategy introduced by Bruchon et al. in [4] [15].
Indeed, as P1 description of the LS is considered in the proposed methodology, one of the basic issues in the problem of surface diffusion is that the velocity is defined by the Laplacian of the curvature, which means that the velocity is a function of the fourth order spatial derivative of $\varphi$. The numerical strategy proposed by Bruchon et al. consists to solve this problem by considering a "regularized" formulation. More precisely Eq.(18) is solved in a weak form by using a FE formulation. Further informations can be found in [4] [15].

\subsection{Convection-Reinitialization methodology}

By assuming the appropriate calculation of the surface velocity, the traditional strategy of convection and subsequent reinitialization steps is used. The main idea is to solve the advection equation and to rebuild the metric properties of the level-set function in order to keep a distance function $(\|\nabla \varphi(x, t)\|=1)$ at least near the interface $\Gamma(t)$. Classical approaches consist in solving, separately, the convective part and the reinitialization part thanks to the resolution of a classical Hamilton-Jacobi system [11] or to adopt an unified advection and renormalization methodology by solving one single equation based on a smooth description of the level-set [15]. Here, a new approach is proposed.

This new approach is based on a separate resolution of the transport and of the reinitialization part. Convective equation is firstly solved thanks to a stabilized P1 solver (SUPG or RFB method). Then, a parallel and direct reinitialization algorithm detailed in [16], which has been proven to be extremely fast and accurate is used. In this algorithm, the $\Gamma(t)$ interface is firstly discretized into a collection of segments (respectively triangles in 3D) and the nodal values of the level-set function are then updated by finding the nearest element of the collection and calculating the distance between the considered node and this nearest element. This method takes advantage of a space-partitioning strategy using $k$ - $d$ tree and an efficient bounding box strategy enabling to maximize the numerical efficiency for parallel computations.

Moreover, this methodology presents two other interest:

-it enables to avoid the validation/calibration of unphysical parameters necessary to reinitialize [11] [15],

-it enables to obtain directly an exact P1 description of $\boldsymbol{n}$ [17] before to solve Eq. (18) rather than following the classical less precise methodology where the normal is computed by performing a P1 interpolation of the first derivative of the level-set function. 


\section{First academic case}

In this section we examine an ellipsoid shape under surface diffusion. We want to test the efficiency of the proposed formulation for a simple case with an analytical solution. Considered computational domain is a $1 \mathrm{~mm} \times 1 \mathrm{~mm}$ square centered in $(0,0)$. An initial ellipse $(a=0.3 \mathrm{~mm}$ and $b=0.2 \mathrm{~mm})$ of equation $x^{2} / a^{2}+y^{2} / b^{2}=1$ is considered. Of course, this shape is going to evolve towards a circle shape while conserving its area. Thus limit radius, i.e. limit value of $a$ and $b$ is given by the value $\sqrt{\pi a b} \approx$ $0.43416 \mathrm{~mm}$.

Initially, in order to test the efficiency of the approach without dealing with the effect of the meshing and remeshing in the results, a fixed mesh is considered during simulations. An initial isotropic mesh adaptation is considered in a ring centered in $(0,0)$ and defined as $0.19 \mathrm{~mm} \leq r \leq 0.31 \mathrm{~mm}$ in order to keep a very fine mesh, defined as $h$ in Table 1 , in all the zone crossed by the zero isovalue of the levelset function during the simulation.

The figure 3 shows the FE mesh used (a), the initial distance function field (b), a zoom on the FE mesh (c), the curvature field near the interface (d) and the normal velocity field near the interface obtained initially thanks to the FE resolution of Eq. (18) (e). Red or white line corresponds to the initial ellipse interface ( 0 isovalue of the level-set function).

In all simulations $B$ is assumed to be homogeneous and equal to $1 \mathrm{~mm}^{4} / \mathrm{s}$. Exact velocity of the point $(a(t), 0)$ is known [15] and given by:

$$
\overrightarrow{v_{a}}(t)=B \frac{3\left(b^{2}-a^{2}\right) a}{b^{6}} \vec{\imath}=v_{a}(t) \vec{\imath} .
$$

Hence $a(t+d t)$ can be easily evaluated thanks to a forward Euler method:

$$
a(t+d t)=a(t)+d t v_{a}(t)
$$

and $b(t+d t)$ can be easily obtained by verifying the area conservation at anytime:

$$
b(t+d t)=a(0) b(0) / a(t+d t) .
$$

This method is then used with a time step of $1 \mathrm{~ms}$ to evaluate the "exact" evolution of $a$ and $b$ values during the shape evolution. Concerning FE simulations, at each time step, the positions of $(a(t), 0)$ and $(0, b(t))$ are determined on the zero-isovalue of the distance function and then compare to the "exact" solution. Final time of all simulations is fixed to $1 s$.
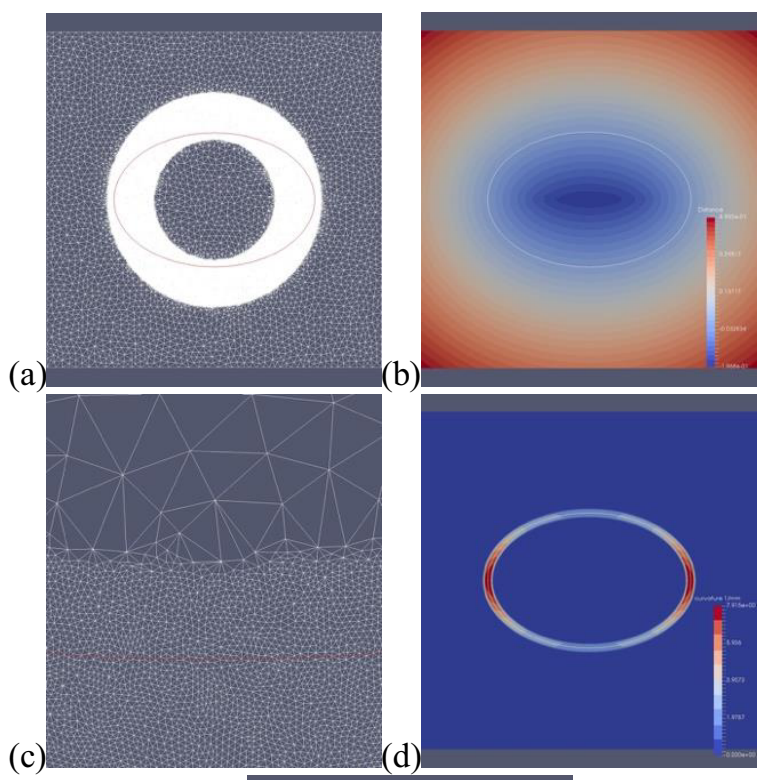

(e)

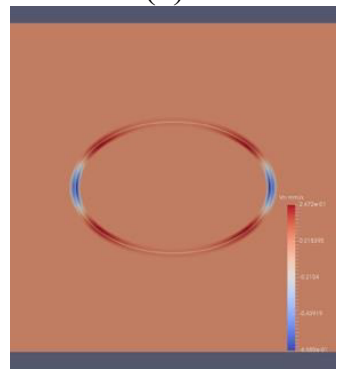

Figure 3. First academic case: the FE mesh used (a), the initial distance function field (b), zoom on the FE mesh at the interface between coarse and fine mesh (c), $\boldsymbol{\kappa}$ near the interface (d) and $\boldsymbol{v}_{\boldsymbol{n}}$ near the interface (e). Red or white line corresponds to the initial ellipse interface (zero-isovalue of the level-set function).

Table 1 summarizes all the parameters (time step, mesh size in the fine mesh zone, number of elements of the used mesh, method used), the CPU time of the simulations and the corresponding precision of the results obtained concerning the positions of $(a(t), 0)$ for $t \in[0,1 s]$ by using the unified convectiverenormalized approach described in [15] and the new one proposed here with different time step and $h$ values. These cases are representatives of an important number of other performed simulations.

Errors are defined as:

$$
\begin{gathered}
e_{1}=\frac{\left\|a_{\text {sim }}(t)-a_{\text {exact }}(t)\right\|_{L_{1}}}{\left\|a_{\text {exact }}(t)\right\|_{L_{1}}}=\frac{\sum_{i}\left|a_{\text {sim }_{i}}-a_{\text {exact }_{i}}\right|}{\sum_{i}\left|a_{\text {exact }_{i}}\right|} \text { and, } \\
e_{2}=\frac{\left\|a_{\text {sim }}(t)-a_{\text {exact }}(t)\right\|_{L_{2}}}{\left\|a_{\text {exact }}(t)\right\|_{L_{2}}}=\frac{\sqrt{\sum_{i}\left(a_{\text {sim }_{i}}-a_{\text {exact }}\right)_{i}}}{\sqrt{\sum_{i} a_{\text {exact }}^{2}}},
\end{gathered}
$$

where $i$ denotes the discretization in time. 
Table 1. Summarized data and results of some tested configurations

\begin{tabular}{|c|c|c|c|c|}
\hline Data & Case1 & Case2 & Case3 & Case4 \\
\hline$h$ in fine zone in $\mu m$ & 1 & 1 & 2 & 1 \\
\hline \#Elt & $2,74 \mathrm{e} 5$ & $2,74 \mathrm{e} 5$ & $7,1 \mathrm{e} 4$ & $2,74 \mathrm{e} 5$ \\
\hline Time step in $m s$ & 1 & 5 & 10 & 0.1 \\
\hline Conv + exact Reinit & $\mathrm{X}$ & $\mathrm{X}$ & $\mathrm{X}$ & \\
\hline Unified approach & & & & $\mathrm{X}$ \\
\hline Calculation time on 12CPU & $1 \mathrm{~h}$ & $17 \mathrm{mins}$ & $1 \mathrm{~min}$ & $6 \mathrm{~h} 28 \mathrm{mins}$ \\
\hline $\mathrm{e} 1$ in $\%$ & 2.5 & 2.6 & 2.1 & 2.2 \\
\hline $\mathrm{e} 2$ in $\%$ & 3 & 3.1 & 2.9 & 2.8 \\
\hline
\end{tabular}

From different simulations (Cases 1 to 4 of Table 1 are representatives), we can summarize the following comments:

-Considering the precision of $a(t)$ predictions (see $e_{1}$ and $e_{2}$ errors on Table 1), both approaches are relevant to model surface diffusion. Figure 4 illustrates at $t=1 \mathrm{~s}$, the difference of the exact interface and the results obtained with the Case1.

-The time step of the Case $4(d t=0.1 \mathrm{~ms})$ is the maximal value usable for $h=1 \mu m$ and the unified formulation to avoid numerical instabilities. Such instabilities were not identified for the new proposed approach even for important time step and mesh size (Case 3 for example).

-As illustrated in Table 1, calculation time of the new proposed approach is then clearly very attractive comparatively to the unified approach.

-Even for the new proposed approach, decreasing the $d t$ and $h$ values below, respectively, $10 \mathrm{~ms}$ and $2 \mu \mathrm{m}$ seems not improve the results quality $\left(e_{2} \approx\right.$ $3 \%)$. It can be explained by the fact that the residual error is due to the FE resolution of Eq. (18).

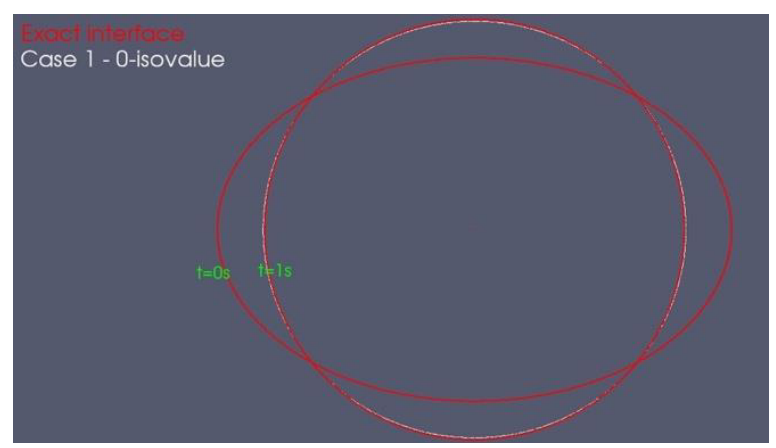

Figure 4. Comparison at $t=0 s$ and $t=1 s$ of the exact solution (red lines) and the Case 10 -isovalue (white lines).

\section{Second academic case: volume loss and mesh adaptation}

Next, we consider a more realistic shape of a long ellipse with $a=0.5 \mathrm{~mm}$ and $b=0.1 \mathrm{~mm}$ and mesh adaptation. Indeed, in order to propose acceptable calculation time to model surface diffusion of complex microstructure such as one of the Figure 1, fixed meshing strategy of the previous section is not an option. Thus a meshing and remeshing strategy must be used.

To begin, two based-metric meshing strategies associated with the MTC topological mesher were tested. MTC is a P1 automatic remesher based on elements topology improvement that was developed for Lagrangian simulations under large strains. This tool was extended to anisotropic mesh adaptation, for which it was extensively used in context of FE microstructure description [11] [10] [14] [15] [4] [17].

The first metric considered is the metric describes in [14], this metric enables to impose isotropic or anisotropic (in the normal direction of the interface) fine mesh in the vicinity of the interface without considering its curvature. Here, isotropic adaptation was considered (Method 1 in Table 2).

The second used metric field is based on an a priori error estimator linking the interpolation error on the LS function to its gradient vector and hessian matrix. As already described, these variables represent respectively the normal vector to the interface and its main curvatures. Using this data, a metric field can be built in order to have a very fine mesh size in the normal direction to the interface, and to control the mesh size in the other directions depending on local curvature [18] (Method 2 in Table 2).

Simulation and data results are reported in Table 2 for both meshing/remeshing methods. The "Conv + exact Reinit" strategy was used. Final time of both simulations is fixed to $1 s$. As the mesh size near the interface is of the same order than the mesh size used in Case1 and Case2, same precision could be expected. However, remeshing is also synonymous of diffusion concerning the FE fields carrying the interface at each remeshing operation necessary to follow interface motion. Then, volume conservation was tracked for these cases. 
Table 2. Summarized data and results with mesh adaptation.

\begin{tabular}{|c|c|c|}
\hline Data & Case5 & Case6 \\
\hline$h \_$min in the fine zone in $\mu m$ & 1 & 1 \\
\hline \#Elt & $<10000$ & $<10000$ \\
\hline Time step in $m s$ & 1 & 1 \\
\hline Conv + exact Reinit & $\mathrm{X}$ & $\mathrm{X}$ \\
\hline Remeshing period & $5 \mathrm{dt}$ & $5 \mathrm{dt}$ \\
\hline Remeshing method & Method 1 & Method 2 \\
\hline Calc. Time on 12CPU & 7 mins & 4 mins \\
\hline Volume loss in \% & 7.5 & 1 \\
\hline
\end{tabular}

Results described in Table 2 illustrate that the mesh adaptation technique come with good precision and faster calculation time. Meshing adaptation based on the curvature enables to obtain a very good conservation of the volume. This aspect will be of course very important for real configurations where the initial thin shape of the $\alpha$ lamellae implies very high ratio between the minimal and the maximal values of the interface curvature. So, this meshing strategy in terms of metric seems particularly indicated.

\section{Discussions}

If the results described previously in terms of calculation time and precision could be sufficient to study the surface diffusion of one thin ellipse, it seems clear that to further improve our numerical framework, it is important to deal with real $2 \mathrm{D}$ or $3 \mathrm{D}$ configurations.

In order to face this problem, we adopt a new topological mesher, Fitz, developed by Shakoor et al. [18]. With Fitz, a body fitted meshing and remeshing is possible while using complex metric as previously described. It was then proved in [19] that this new mesher associated with a volume conservation constraint which is compatible both with implicit and body-fitted interfaces, the modification of the interface due to remeshing can be delayed enough so that a Lagrangian Level-Set method becomes more than interesting compared to an Eulerian Level-Set method, even when large deformations or displacements occur.

First tests are very promising, allowing in the Case6 configuration to obtain a precision of $2 \%$ concerning the volume conservation with a calculation time of $30 \mathrm{~s}$ on $12 \mathrm{CPU}$. Figure 5 illustrates a result obtained with this numerical framework for a " $a=0.5 \mathrm{~mm}$ and $b=0.05 \mathrm{~mm}$ " configuration.

Moreover we anticipate with this mixed implicit/explicit description of the interfaces an easier coupling between surface diffusion at the $\alpha / \beta$ interfaces and motion by mean curvature at the $\alpha / \alpha$ grain interfaces.

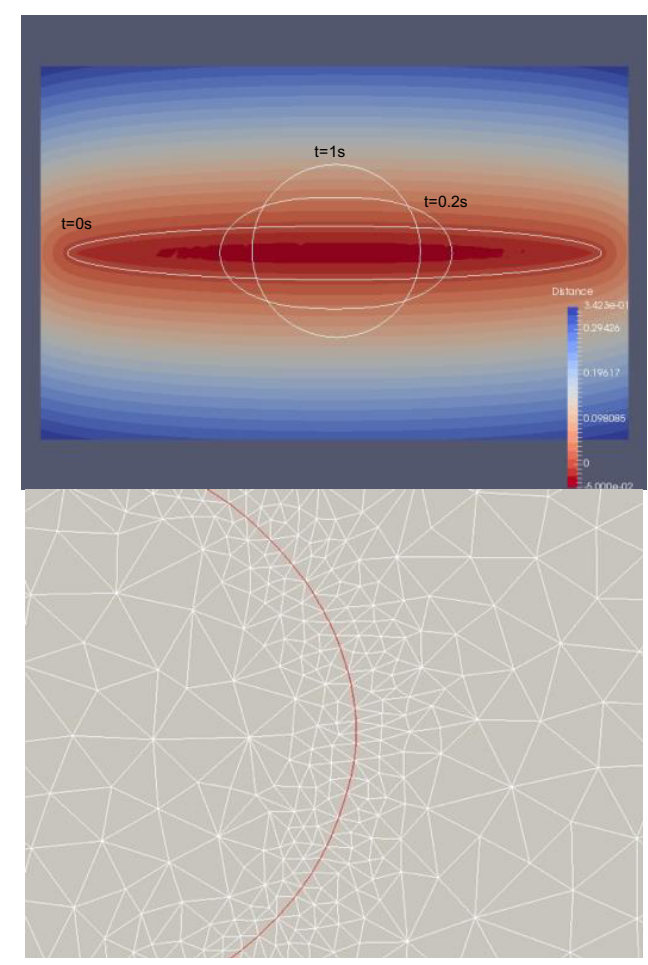

Figure 5. A " $a=0.5 \mathrm{~mm}$ and $b=0.05 \mathrm{~mm}$ " configuration with a mixed implicit/explicit description of the interfaces: (top) initial distance function field and the obtained interfaces (white lines) at $\mathrm{t}=0 \mathrm{~s}, 0.2 \mathrm{~s}$ and $1 \mathrm{~s}$. (Bottom) Zoom on the conforming mesh at $\mathrm{t}=0.2 \mathrm{~s}$, the interface is defined by the red line.

\section{Conclusions}

The first steps of a new FE numerical framework dedicated to the modelling of the mechanisms of spheroidization in $\alpha / \beta$ titanium alloys have been detailed. This numerical framework has been illustrated on the surface diffusion of $\alpha$ laths. Several cases were studied in order to check the efficiency of the proposed approach. Optimal algorithm of resolution and meshing strategies were proposed in terms of precision and calculation time. A new way was also opened by considering a mixed implicit/explicit description of the interfaces. All this numerical tools will now be exploited to deal with coupling between surface diffusion at the $\alpha / \beta$ interfaces and motion by mean curvature at the $\alpha / \alpha$ grain interfaces. An existing crystal plasticity finite element approach developed in a level-set context [20] [17] will also be used to model the deformation of the $\alpha$ lamellae. 


\section{Acknowledgements}

The authors would like to gratefully thank Aubert \& Duval, CEA, Timet and Safran for funding this research through the SPATIALES consortium.

\section{References}

1. G. Lütjering, J. Williams, Titanium (Springer: Heidelberg, 2007)

2. S. Semiatin, D. Furrer, Metals Branch (Metals, Ceramics, and NDE Division, 2008)

3. W. Mullins, Acta Metallurgica 6, 414-427 (1958)

4. J. Bruchon, D. Pino Muñoz, F. Valdivieso, S. Drapier, G. Pacquaut, European Journal of Computational Mechanics 19, 281-292 (2010)

5. V. Derkach, M.Sc. thesis, Department of Mathematics, Technion-Israel Institute of Technology (2010)

6. V. Derkach, A. Novick-Cohen, A. Vilenkin, E. Rabkin, Acta Materialia 65, 194-206 (2014)

7. P. Smereka, Journal of Scientific Computing 19, 439-456 (2003)

8. S. Osher, F. Fedkiw, Journal of Computational Physics 169, 463-502 (2001)

[9] J. Sethian, Level Sets Methods and Fast Marching Methods (Cambridge Monograph on Applied and Computational Mathematics, 1999)

10. M. Bernacki, R. Logé, T. Coupez, Scripta Materialia 64, 525-528 (2011)

11. M. Bernacki, Y. Chastel, T. Coupez, R. Logé,

\section{Scripta Materialia 58, 1129-1132 (2008)}

12. M. Burger, F. Hausser, C. Stocker, A. Voigt, Journal of Computational Physics 225, 183-205 (2007)

13. H. Digonnet, L. Silva, T. Coupez, in Proceedings of the 9th International Conference on Numerical Methods in Industrial Forming Processes (2007)

14. M. Bernacki, H. Resk, T. Coupez, R. Logé, Modelling and Simulation in Materials Science and Engineering 17, 064006 (2009)

15. J. Bruchon, S. Drapier, F. Valdivieso, International Journal for Numerical Methods in Engineering 86, 845-861 (2011)

16. M. Shakoor, B. Scholtes, P.-O. Bouchard, M. Bernacki, Applied Mathematical Modelling 39, 7291-7302 (2015)

17. B. Scholtes, R. Boulais-Sinou, A. Settefrati, D. Pino Muñoz, I. Poitrault, A. Montouchet, N. Bozzolo, M. Bernacki, Computational Materials Science 122, 57 71 (2016)

18. M. Shakoor, M. Bernacki, P.-O. Bouchard, Engineering Fracture Mechanics 147, 398-417 (2015)

19. M. Shakoor, P.-O. Bouchard, M. Bernacki, International Journal for Numerical Methods in Engineering (In press)

20. H. Resk, L. Delannay, M. Bernacki, T. Coupez, R. Logé, Modelling and Simulation in Materials Science and Engineering 17, 075012 (2009) 